\title{
Surface Sensitive and Compositional SEM Imaging for High Accelerating Voltages in Focused Ion/Electron Beam Systems
}

\author{
Toshihide Agemura*, Tsunenori Nomaguchi* and Tsuyoshi Onishi*
}

* Advanced Microscope Systems Design $2^{\text {nd }}$ Department, Naka Division, Nanotechnology Products Business Group, Hitachi High-Technologies Corporation, Hitachinaka, Ibaraki 312-8504, Japan

It is very common to use accelerating voltages from $0.1 \mathrm{kV}$ to $30 \mathrm{kV}$ in scanning electron microscopes (SEM) and to collect secondary electrons (SE) for imaging applications. The SE signal consists of electrons (SE1) produced by primary electrons (PE) and those (SE2) excited by backscatter electrons (BSE). The escape depth of SE1 is shallow on the order of nms, so the SE1 have information about the surface of a specimen. On the other hand, the escape depth of SE2 is roughly half of the PE interaction volume and therefore the SE2 have information about the inner part of the specimen. Since the mean energy of the BSE is lower than the PE energy, and the emergence angle of the BSE is lower than the normal incidence angle of the PE, the BSE produce more SE than the PE. This means the yield ratio of SE2 to SE1 is typically much more than unity; therefore, almost all SE detected in the SEM reflects the inner information of the specimen. To increase the surface sensitivity of SE imaging at lower accelerating voltages are used to shrink the size of the interaction volume between the PE and the specimen. On the other hand, the beam probe size becomes larger theoretically as decreasing the accelerating voltage. A retarding method [1] and a boosting method [2] have been introduced to conventional SEMs in order to improve the resolution at the lower accelerating voltages. However, especially in the focused ion/electron beam system, specimens are thin and very small and have to be set at a beam cross point where the ion beam is coincident with the electron beam and the working distance is usually more than $4 \mathrm{~mm}$ on the SEM axis. These conditions are drawbacks against enabling the retarding and boosting methods effectively.

Elastically and plurally scattered electrons in the BSE are categorized into low-loss electrons (LLE). The LLE suffer only small number of scattering inside the specimen so that the penetration of the PE is very shallow and not only the material information but also the surface sensitive information can be obtained by detecting the LLE even at higher accelerating voltages with keeping smaller probe size than that at lower accelerating voltages. Wells is a pioneer and has researched on the LLE technique for many years [3-10]. Cross-sectional SEM images of a DRAM specimen are shown in FIG. 1. The specimen was fabricated by the Hitachi NB5000 focused ion/electron beams system as exposing and not exposing capacitor trenches on the same surface. FIG. 1(a) shows a SE image taken with an ET detector. FIG. 1(b) and (c) show the LLE images acquired with 1\% and 2\% energy windows respectively. Both images were taken at $20 \mathrm{kV}$ with a $80 \mathrm{~s}$ frame speed. The LLE detector consists of two semi-spherical energy grid filters attached to the other ET detector. The entrance grid and the scintillator surface of the ET detector were kept at ground potential and the filter grid was at negative $19.8 \mathrm{kV}$ and $19.6 \mathrm{kV}$ relative to the ground potential so the energy windows were $0.2 \mathrm{~V}$ and $0.4 \mathrm{kV}$. As predicted, it is obvious that the depth information becomes shallow as narrowing the potential windows and the contrast depending on materials inside the DRAM becomes darker as dwindling the atomic and/or molecular mass. In order to check the compositional contrast more clearly, SEM images of a $\mathrm{Zr} / \mathrm{TiC} / \mathrm{Al}_{2} \mathrm{O}_{3}$ specimen were obtained. FIG. 2(a) shows a SE image at $20 \mathrm{kV}$ using a 80s frame speed and FIG 2(b) shows a LLE image with the 1\% energy 
window using a $320 \mathrm{~s}$ frame speed. The both images were taken at $20 \mathrm{kV}$. The compositional contrast with respect to the atomic or molecular mass and even the small difference of contrast on the same material of TiC can be clearly seen in the LLE image of FIG. 2(b). Moreover, the charging contrast due to the SE on the SE image of FIG. 2(a) is disappeared in the LLE image. The major drawback of LLE detection is the longer acquisition times required to obtain sufficient signal, which may not be suitable for applications where the throughput is very important. However, the authors believe that the LLE including the surface and compositional information with the small probe size are still informative and are well suited for many applications in the focused ion/electron beam systems because of the particular conditions as discussed above.

\section{References}

[1] R. F. W. Pease, IEEE 9th Annual Symposium on Electron, Ion and Laser Beam Technology, 176-187 (1967).

[2] J. Frosien et al., J. Vac. Sci. Tehnol. B7(6), 1874-1877 (1989).

[3] O. C. Wells, Appl. Phys. Lett. 16(4), 151-153 (1970).

[4] O. C. Wells, Appl. Phys. Lett. 19(7), 232-235 (1971).

[5] O. C. Wells et al., Appl. Phys. Lett. 23(6), 353-355 (1973).

[6] O. C. Wells, Scanning 2, 199-216 (1979).

[7] O. C. Wells, Scanning 8, 120-126 (1986).

[8] O. C. Wells et al., Appl. Phys. Lett. 56(23), 2351-2353 (1990).

[9] M. T. Postek et al., Scanning 23, 298-304 (2001).

[10] O. C. Wells et al., Scanning 23, 366-371 (2001).
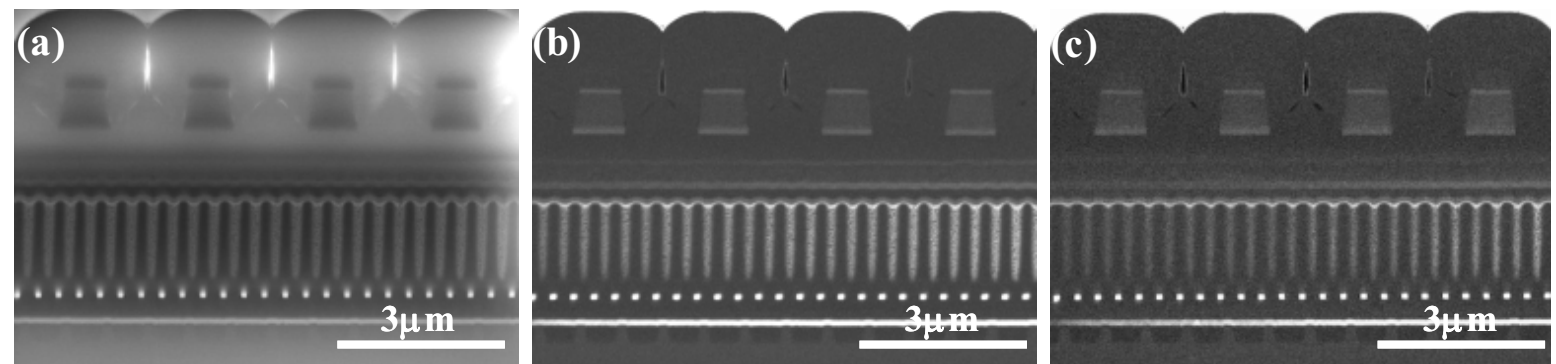

FIG. 1. The SE images of a DRAM device. The field of width of the images is $10 \mu \mathrm{m}$. All images were taken at $20 \mathrm{kV}$ with $80 \mathrm{~s}$ acquisition time. The SE image (a) shows the inner information of the device. The LLE images that the potential windows were set at $1 \%$ (b) and $2 \%$ (c) positive to the $-20 \mathrm{kV}$ give the surface and the compositional information.
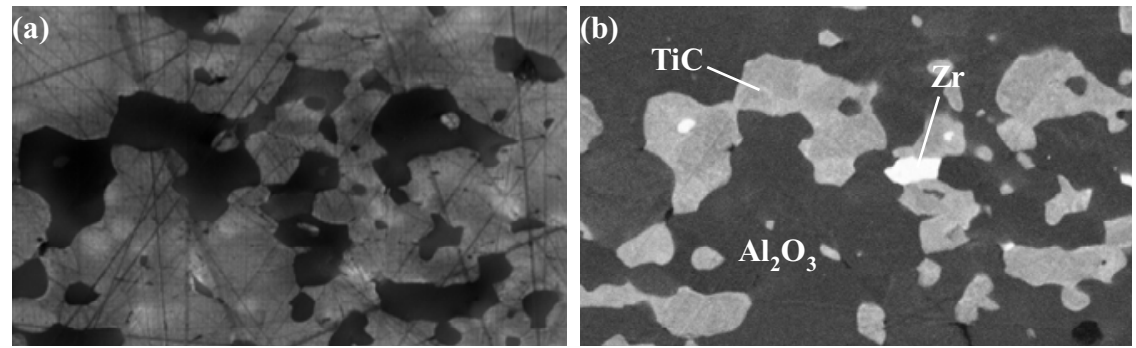

FIG. 2. The SE images of a $\mathrm{Zr} / \mathrm{TiC} / \mathrm{Al}_{2} \mathrm{O}_{3}$ material. The field of width of the images is $8.5 \mu \mathrm{m}$. All images were taken at $20 \mathrm{kV}$. The SE image (a) taken with 80 s acquisition time does not show the material contrast but the charging contrast due to the SE detection. The 1\% LLE image (b) taken with 320s acquisition time clearly gives the compositional contrast even on the same material of TiC. 